\title{
Opisthorchiasi autoctona al Lago Trasimeno (Perugia): descrizione di due episodi epidemici da Opisthorchis felineus e problematiche diagnostiche differenziali
}

\author{
Daniele Crotti', Maria Letizia D'Annibale ${ }^{2}$, Silvia Crotti ${ }^{3}$ \\ 'Libero Professionista in Parassitologia e Microbiologia Medica, Perugia \\ ${ }^{2}$ Struttura Complessa di Microbiologia, Azienda Ospedaliera, Perugia \\ ${ }^{3}$ Istituto Zooprofilattico Sperimentale dell'Umbria e delle Marche, Perugia
}

Key words: opisthorchiasis, Opisthorchis felineus, intestinal parasitosis, tench, Umbrie

Detection of human opisthorchiasis in the Trasimeno Lake area (Perugia, Italy): two authoctonous clusters caused by Opisthorchis felineus.

\section{SUMMARY}

The Authors describe two small and apparently asymptomatic epidemics of Opisthorchiasis caused by Opisthorchis felineus, which were observed in provence of Perugia, Umbrie region, Italy. The first one was observed in a couple of young adults during 2003 because both ones ate pickled (at 2-8 ${ }^{\circ} \mathrm{C}$ ) tenches fished in Trasimeno Lake in province of Perugia. The second episode was observed during 2006 among eight young adults that ate pickled fishes (tenches, carps and perches, always at $2-8^{\circ} \mathrm{C}$ ); source of these fishes was the same lake. The request for a coproparasitological examination of stools in one of the two subjects of the first episode and in one of the eight subjects of the second episode was the casual observation of an increased count of periferical eosinophils. During January 2006, ten faecal samples of cats, living on "Isola Maggiore" of the Trasimeno Lake, were collected for a parasitological test; $40 \%$ was positive for ova of $O$. felineus. The Authors describe the diagnostical items for this parasitosis, relate the epidemiological features of this trematode infection, and suggest the importance of a good approach to this zoonotic parasitosis, which interest public health too and never were before observed in Italy among humans.

\section{INTRODUZIONE}

Opisthorchis felineus è un trematode, il cui adulto ermafrodita, che misura 7-12 $\mathrm{mm}$ di lunghezza $\mathrm{x}$ 2-3 mm di larghezza, alberga i dotti biliari intraepatici di felidi e canidi (rappresentando il gatto l'ospite definitivo principale); l'uomo è ospite definitivo secondario, nel quale, in seguito ad ingestione accidentale di metacercarie, si sviluppa l'opisthorchiasi (9). Le uova, eliminate in acque dolci dagli animali infestati, vengono ingerite da un piccolissimo mollusco (la lumaca Bythinia leachi, che rappresenta il I ospite intermedio), ove si schiudono liberando il miracidio, che matura a sporocisti, poi a redia ed infine a cercaria (8). Tale lofocercaria, rilasciata dopo circa 2-3 mesi dal mollusco in questione, penetra attivamente nella muscolatura di un pesce d'acqua dolce (tinca ed altri, quali barbo e carpa principalmente), che rappresenta il II ospite intermedio, in cui si trasforma in metacercaria (11). L'ospite definitivo si infesta ingerendo i pesci crudi (o malcotti o marinati a freddo) con le metacercarie, che raggiungono poi i dotti biliari intraepatici risalendo per via canalicolare dal duodeno; in tali canali biliari maturano ad adulti ed ivi depongono le uova (10). Il periodo di prepatenza è stimato in 3-4 settimane (8).

A differenza di altri trematodi molto simili (tabella 1), che sono presenti esclusivamente in alcuni Paesi dell'Estremo Oriente (Opisthorchis viverri$n i$, Clonorchis sinensis) ed anche in Europa dell'Est e/o aree mediterranee (Metagonimus yokogaway, Heterophyes heterophyes), O. felineus ha una distribuzione geografica differente (tabella 2). E' infatti segnalato sia in Siberia e Russia, sia nell'Europa dell'Est e balcanica, sia in Germania e, verosimilmente, anche in Spagna ed Italia, quanto meno in campo animale $(2,15,16)$. Esistono altre due specie del genere Heterophyes così come tre specie del genere Haplorchis, assai simili ai precedenti, che possono interessare l'uomo; ma le infestazioni da questi sostenute sono talmente rare o eccezionali che verranno omessi del tutto in questa presentazione (3).

Mentre gli adulti di $O$. felineus, di $O$. viverrini e di $C$. sinensis si localizzano essenzialmente nei dotti biliari intraepatici, per gli adulti di $H$. heterophyes e di $M$. yokogaway la sede di localizzazione principale è il piccolo intestino (9).

La diagnostica si basa sulla attenta ricerca delle piccole uova, in microscopia ottica, da più cam- 
pioni fecali dei soggetti infestati, utilizzando preferibilmente la tecnica di concentrazione formoloetere/etilacetato (FEA) (4). Va peraltro sottolineato come sia assai difficile riconoscere le differenze tra le uova di Opistorchis/Clonorchis e quelle di Heterophyes/Metagonimus, essendo le medesime molto simili; ed ancora più improba è la distinzione delle specie all'interno dei due gruppi citati (tabella 3). Ne consegue che solitamente la diagnosi di certezza si basa sul supporto dei dati epidemiologici e/o con lo studio dei vermi adulti, quando possibile analizzarli, in quanto o prelevati dopo un intervento chirurgico o liberati con le feci, in seguito a somministrazione di un purgante e/o del farmaco anti-elmintico specifico $(7,9)$.

$O$. felineus non è mai stato segnalato nell'uomo nel nostro Paese. Soltanto molti decenni addietro le uova di tale trematode sono state segnalate in gatti e cani $(12,13)$. Riportiamo così le prime segnalazioni relative a due episodi epidemici di opisthorchiasi, verosimilmente sostenuti, appunto, da O. felineus.

\section{MATERIALI E METODI \\ Descrizione del I episodio epidemico}

In data 14 luglio 2003 un soggetto maschio di 42 anni, di nazionalità italiana e residente in Perugia, viene ricoverato in un reparto di Medicina Interna dell'Azienda Ospedaliera di Perugia, in quanto affetto da tempo da periodico rialzo termico e cefalea frontale, con nausea e marcata astenia. Durante il ricovero viene riscontrata una ipereosinofilia periferica (14\%); nel sospetto, o comunque per escludere una parassitosi intestinale, viene richiesto un esame coproparassitologico. La visita clinica è negativa e le indagini ematochimiche mostrano tutte i parametri nella norma eccezion fatta per la VES (23 alla I ora [valore di riferimento: < 16]) e per le gamma-GT $(81 \mathrm{mU} / \mathrm{ml}$ [valore di riferimento: $<50 \mathrm{mU} / \mathrm{ml}$ ]).

Sul primo campione (del 15 luglio) viene eseguito un esame coproparassitologico standard (ECPS): osservazione diretta macroscopica e microscopica (feci formate, non leucocitosi fecale né ematochezia), osservazione microscopica dopo FEA (con risultato negativo) e colorazione di Giemsa (4). In questo campione, che verrà congelato a $-20^{\circ} \mathrm{C}$, viene evidenziata la presenza di numerosi trofozoiti di Dientamoeba fragilis (5). All'indagine anamnestica non risultano apparentemente fattori di rischio particolari, sebbene il soggetto, commerciante di professione, abiti in zona rurale e possieda un cane di compagnia: beve infatti solo acqua minerale, non utilizza acqua di pozzo per lavare frutta e verdura, non riferisce cure termali con fanghi (recenti o pregresse), non è mai stato in Paesi in via di svilup- po (PVS). Un secondo ed un terzo campione fecale confermano quanto sopra osservato.

Vengono condotti successivamente altri vari accertamenti, tutti ad esito negativo, mentre un'ecografia addominale mostra un fegato lievemente aumentato di volume, con incremento omogeneo e diffuso dell'ecodensità parenchimale (steatosi epatica di lieve grado); tutto il resto è invece nella norma.

In data 18 luglio il soggetto viene dimesso con la diagnosi di "Parassitosi intestinale da Dientamoeba fragilis". La terapia consigliata è con paromomicina (Humatin: $2 \mathrm{cp}$ da $250 \mathrm{mg}, 3$ volte al giorno per 6 giorni), con il suggerimento di eseguire un controllo dopo almeno 2 settimane dalla fine della terapia. Le altre indagini in corso al momento della dimissione (dosaggio IgE, tipizzazione linfocitaria, marcatori autoimmunità, complesso TORCH, Widal-Wright, sierodiagnosi per idatidosi e markers dell'epatite virale) risulteranno tutte negative.

In data 14,18 e 20 agosto il soggetto esegue i tre ECPS di controllo. In tale occasione il soggetto riferisce la scomparsa dei disturbi che lo avevano costretto al ricovero, permanendo solamente un vago "senso di stanchezza". L' ECPS mette in evidenza la persistenza di $D$. fragilis e la presenza di forme vacuolate di Blastocystis hominis, nonché la presenza di rare uova attribuibili al gruppo Clonorchis/Opistorchis (vedi tabella 4). Il soggetto viene allora richiamato per ulteriori ragguagli. Questi ritorna, dopo le ferie estive, a fine settembre; vengono analizzati i campioni fecali raccolti nei giorni 26 e 27 settembre, e $1^{\circ}$ ottobre. Si riconferma la presenza di $D$. fragilis (per la quale si prescrive terapia con paromomicina per 7 giorni con dosaggi giornalieri di $30 \mathrm{mg} / \mathrm{kg}$ di peso corporeo in 3 dosi), di $B$. hominis, e di piccole uova percolate identificate come uova di Opistorchis felineus (figura I). Tale diagnosi viene fatta sull'attenta disamina delle uova (vedi tabella 3) e, soprattutto, su un'attenta valutazione epidemiologico-anamnestica (11). Il soggetto infatti nega qualsivoglia viaggio all'estero (in particolare in PVS e Paesi dell'est Europa e in Asia), così come di avere mai mangiato in ristoranti esotici con cucine a base di pesce anche non cotto (15). Riferisce invece di aver consumato, in data 17 giugno 2003, una porzione di tinca marinata (a freddo) in un ristorante attorno al lago Trasimeno (provincia di Perugia), tinca di provenienza dal lago medesimo (6); il soggetto riferisce inoltre di non avere mai mangiato in precedenza pesce d'acqua dolce (10). Valutando in inferiore al mese il periodo di prelatenza dell'opisthorchiasi, il primo campione a suo tempo congelato viene scongelato per una verifica dell'eventuale presenza già allora (14 luglio, 27 giorni dopo il pasto a 
base di tinca marinata) di tali uova. Ebbene, dopo una concentrazione FEA del campione scongelato, al quinto preparato microscopico (osservato a $10 \times 10$ e quindi $40 \times 10$ ) viene ritrovato un uovo ben conservato di $O$. felineus.

Dal momento che ad una ripetuta domanda, la risposta è quella che anche la moglie aveva assaggiato la porzione di tinca "incriminata", viene richiesto un campione fecale della signora. L'ECPS mette in evidenza (dopo FEA) anche nella moglie uova, sia pur rare, di $O$. felineus.

Il referto finale è quello di una opisthorchiasi asintomatica nella coppia, per cui si decide di soprassedere al momento e non procedere a terapia specifica (nel frattempo si era negativizzata la presenza di $D$. fragilis).

\section{Descrizione del II episodio epidemico}

Ai primi di gennaio 2006, ad un soggetto maschio di 29 anni viene prescritta un ECPS in quanto ad un esame emocromocitometrico di controllo persiste una ipereosinofilia periferica $(9 \%$; valori di riferimento: $<5 \%$ ), già osservata in precedenza nel corso del mese pregresso (riferita peraltro del $20 \%$ ). Il soggetto in questione aveva fatto varie terapie con diversi farmaci antinfiammatori in quanto da tempo sofferente di pubalgia. In tale circostanza il soggetto riferisce anche blandi disturbi intestinali, dallo stesso imputabili ad una non meglio specificata intolleranza alimentare.

L'ECPS viene eseguito su 3 campioni di feci raccolti a giorni alterni; vengono eseguite sia le osservazioni macroscopica e microscopica diretta sia le osservazioni microscopiche dopo concentrazione FEA e dopo colorazione permanente di Giemsa $(4,5)$. L'esito è positivo, e già all'osservazione microscopica diretta, per uova di Opistorchis spp. ( figura I).

Al soggetto viene condotta una accurata anamnesi da cui risulta che il $1^{\circ}$ di novembre 2005 lo stesso con altri sette amici più o meno coetanei aveva consumato pesce marinato a freddo (tinca, carpa, persico) presso un ristorante dell'Isola Maggiore nel Lago Trasimeno, in provincia di Perugia. Vengono così invitati tutti gli altri sette convitati a quel pranzo (4 maschi e 3 femmine) a sottoporsi anche loro a 3 esami coproparassitologici e ad eseguire, se possibile, un conteggio degli eosinofili periferici.

Tra la fine di gennaio e la prima metà di febbraio tutti i soggetti (di età compresa tra 29 e 42 anni) consegnano i rispettivi campioni fecali, che risultano tutti positivi per analoghe uova, sempre attribuibili a Opisthorchis spp. In 3 casi le uova vennero osservate già alla microscopia ottica diretta. In soli 2 casi vennero riferiti i valori del conteggio degli eosinofili, risultati peraltro nei limiti fisiologici.

E' stata contemporaneamente eseguita un'attenta intervista sia al proprietario del ristorante sia al responsabile della Cooperativa Pescatori in località Panicarola di Castiglione del Lago, sempre in provincia di Perugia (e di fatto la medesima di cui al I episodio epidemico), ove parte del pesce (tinche e persici), pescato nel Lago Trasimeno, era stato acquistato (le carpe erano per lo più pescate dallo stesso gestore del ristorante attorno all'Isola Maggiore). Il ristoratore ha affermato di includere nel suo menù culinario "Carpaccio" di pesce di lago, ossia tinca, carpa e persico marinati a freddo (in frigorifero a $2-8^{\circ} \mathrm{C}$ ). Va in ogni caso messo in evidenza come la Cooperativa Pescatori di cui sopra (come per altro tutte le altre esistenti attorno al lago) da sempre raccomanda di consumare adeguatamente cotto il pesce venduto, sia per i dettami di polizia veterinaria vigenti sia per le conoscenze al riguardo circa potenziali rischi di tossinfezione alimentare conseguenti al consumo di pesce pescato non cotto: un proverbio popolare riferito dal responsabile della Cooperativa Pescatori (allo stesso tramandato dai propri avi) recita infatti "pesce cotto, carne cruda" (e non si vuole qui entrare nel merito sulla seconda "inconsueta" affermazione riferita).

Il 29 gennaio 2006 viene fatto un sopralluogo all'Isola Maggiore ove vivono decine di gatti randagi o semi-randagi. Tale isola è una piccola isola di $2 \mathrm{~km}$ di circonferenza, ormai stabilmente abitata da una sola quindicina di persone, soprattutto anziani. Soltanto nei mesi primaverili ed estivi la medesima si affolla per svariate attività turistiche. La presenza di tali gatti randagi o semi-randagi è da tempo nota; questi vengono solitamente alimentati, lungo l'unica via lastricata del piccolissimo borgo, dagli abitanti residenti, con un po' di tutto, da rimasugli o scarti di pesce a crocchette che quotidianamente vengono posti agli angoli della via con i vari anfratti in cui poi i gatti stessi si rintanano al passaggio delle persone. Ivi i gatti defecano e, sempre lungo tale via, sono stati così raccolti dieci campioni, in parte di recente emissione, di deiezioni verosimilmente feline (non esistono cani sull'isola mentre pollame e conigli sono allevati e tenuti in cortili ben protetti).

Le feci feline sono state esaminate al microscopio ottico sia direttamente sia dopo concentrazione FEA: in 4 casi (40\%) sono risultate positive per uova di $O$. felineus (figura II). Le positività sono state osservate sempre già all'esame microscopico diretto ed in due casi il numero di uova rilevate era elevato.

Sulla base pertanto di quanto sinora riferito anche tale episodio epidemico umano è stato attribuito a O. felineus (1). 
Tabella I. Opistorchis spp. e generi correlati

\begin{tabular}{|c|c|c|}
\hline \multicolumn{3}{|c|}{ CARATTERISTICHE PRINCIPALI E DIAGNOSI DIFFERENZIALI } \\
\hline Genere e specie & Etimologia del nome & Famiglia di appartenenza \\
\hline $\begin{array}{l}\text { Clonorchis sinensis } \\
\text { (Opistorchis sinensis) }\end{array}$ & $\begin{array}{l}\mathrm{X} \lambda \omega v=\text { ramoso } \\
\text { Sinensis = cinese }\end{array}$ & Opistorchiidae \\
\hline Opisthorchis felineus & 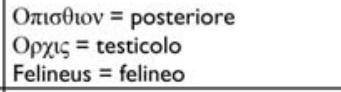 & Opistorchiidae \\
\hline Opisthorchis viverrini & Viverra $=$ un carnivoro selvatico & Opistorchiidae \\
\hline Heterophyes heterophyes & 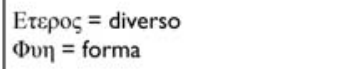 & Heterophyidae \\
\hline Metagonimus yokogawai & $\begin{array}{l}\text { Met } \alpha=\text { posteriore } \\
\text { Гovo }{ }^{\prime} \xi=\text { gonade } \\
\text { Yokogawai = Yokogawa } \\
\text { (parassitologo giapponese) }\end{array}$ & Heterophyidae \\
\hline
\end{tabular}

Tabella 2. Caratteristiche epidemiologiche di Opisthorchis e generi affini.

\begin{tabular}{|c|c|c|c|}
\hline TREMATODE & $\begin{array}{l}\text { I }^{\circ} \text { OSPITE } \\
\text { INTERMEDIO }\end{array}$ & $\begin{array}{c}2^{\circ} \text { OSPITE } \\
\text { INTERMEDIO }\end{array}$ & $\begin{array}{l}\text { DISTRIBUZIONE } \\
\text { GEOGRAFICA }\end{array}$ \\
\hline C. sinensis & $\begin{array}{l}\text { vari molluschi } \\
\text { (lumache) }\end{array}$ & $\begin{array}{l}\text { vari pesci } \\
\text { d'acque dolci }\end{array}$ & $\begin{array}{l}\text { Cina, Corea, Formosa, } \\
\text { Vietnam, Malesia, } \\
\text { Giappone } \\
\text { (India del nord) }\end{array}$ \\
\hline O. felineus & $\begin{array}{l}\text { vari molluschi } \\
\text { (lumache) }\end{array}$ & $\begin{array}{l}\text { vari pesci } \\
\text { d'acque dolci }\end{array}$ & $\begin{array}{l}\text { Polonia, Germania, } \\
\text { Russia, Kazakhistan, } \\
\text { Siberia occidentale } \\
\text { (altri Paesi est-europa?) } \\
\text { (Spagna? Italia?) }\end{array}$ \\
\hline O. viverrini & $\begin{array}{l}\text { vari molluschi } \\
\text { (lumache) }\end{array}$ & $\begin{array}{l}\text { vari pesci } \\
\text { d'acque dolci }\end{array}$ & Tailandia, Laos \\
\hline H. heterophyes & $\begin{array}{l}\text { vari molluschi } \\
\text { (lumache) }\end{array}$ & $\begin{array}{l}\text { vari pesci } \\
\text { d'acque salmastre }\end{array}$ & $\begin{array}{l}\text { Filippine, Formosa, } \\
\text { Corea, Giappone, Cina, } \\
\text { Penisola indocinese, } \\
\text { India, Tunisia, Sudan, } \\
\text { Israele, Iran } \\
\text { (Brasile?) } \\
\text { (altre zone africane ?) }\end{array}$ \\
\hline M. yokogawai & $\begin{array}{l}\text { vari molluschi } \\
\text { (lumache) }\end{array}$ & $\begin{array}{l}\text { vari pesci } \\
\text { d'acque dolci }\end{array}$ & $\begin{array}{l}\text { Giappone, Cina, Siberia, } \\
\text { Filippine, Ucraina, } \\
\text { Azerbajgian, Spagna, } \\
\text { Grecia, Balcani }\end{array}$ \\
\hline
\end{tabular}


Tabella 3. Caratteristiche morfologiche utili per la diagnosi microscopica.

\begin{tabular}{|c|c|}
\hline \multicolumn{2}{|c|}{ MORFOLOGIA DIFFERENZIALE DELLE U O V A } \\
\hline \multicolumn{2}{|c|}{$\begin{array}{l}\text { Le uova hanno tutte una forma ovale, ad "otre", con opercolo ad una estremità; } \\
\text { l'interno è già embrionato (contiene il miracidio); } \\
\text { sono tutte definite di dimensioni piccole, } \\
\text { in quanto sempre inferiori, nella lunghezza massima, ai } 35 \mu \mathrm{m} \text {; } \\
\text { presentano tutte una colorazione gialla o giallo-bruna; } \\
\text { [è difficile, se non a volte impossibile, distinguerle tutte l'una dalle altre; } \\
\text { a volte criteri epidemiologici ed anamnestici possono orientare verso una diagnosi; } \\
\text { la certezza si ottiene solo osservando il verme adulto ermafrodita] }\end{array}$} \\
\hline Clonorchis sinensis & $\begin{array}{l}\text { dimensioni: } 12-19 \times 26-35 \mu \mathrm{m} \text {; } \\
\text { visibile l'opercolo e, sulla estremità opposta, una protuberanza } \\
\text { (ispessimento); } \\
\text { a fianco dell'opercolo è visibile un "orletto": sono margini rilevati (tipo } \\
\text { "due spallette"); } \\
\text { a maggior ingrandimento (rispetto al } 400 \text { ) può essere visibile una } \\
\text { membrana che riveste il guscio. }\end{array}$ \\
\hline Opisthorchis felineus & $\begin{array}{l}\text { dimensioni: } 10-17 \times 28-32 \mu \mathrm{m} \text {; } \\
\text { visibile l'opercolo e, sulla estremità opposta, una protuberanza } \\
\text { (ispessimento) che puó essere anche assente; } \\
\text { I" "orletto" è appena accennato; } \\
\text { - a maggior ingrandimento (rispetto al } 400 \text { ) può essere visibile una } \\
\text { membrana che riveste il guscio; } \\
\text { - usualmente il rapporto lunghezza/larghezza è in media di } 2.75 \text {. }\end{array}$ \\
\hline Opisthorchis viverrini & $\begin{array}{l}\text { dimensioni: } 10-17 \times 19-32 \mu \mathrm{m} ; \\
\text { - visibile l'opercolo e, sulla estremità opposta, una protuberanza } \\
\text { (ispessimento) che può essere anche assente; } \\
\text { - I" "orletto" è appena accennato; } \\
\text { - a maggior ingrandimento (rispetto al } 400 \text { ) può essere visibile una } \\
\text { membrana che riveste il guscio; } \\
\text { - usualmente il rapporto lunghezza/larghezza è in media di I.75. }\end{array}$ \\
\hline Heterophyes heterophyes & $\begin{array}{l}\text { dimensioni : } 14-17 \times 26-30 \mu \mathrm{m} ; \\
-\quad \text { sono leggermente più ovaleggianti; } \\
\text { - visibile l'opercolo; } \\
\text { - l'ispessimento alla estremità opposta è variabile; } \\
\text { l' "orletto" è assente. }\end{array}$ \\
\hline Metagonimus yokogawai & $\begin{array}{l}-\quad \text { dimensioni: } 15-17 \times 26-28 \mu \mathrm{m} ; \\
-\quad \text { sono leggermente più ovaleggianti; } \\
-\quad \text { visibile l'opercolo; } \\
\text { - l'ispessimento all'estremità opposta è variabile; } \\
\text { l' "orletto" è assente. }\end{array}$ \\
\hline
\end{tabular}

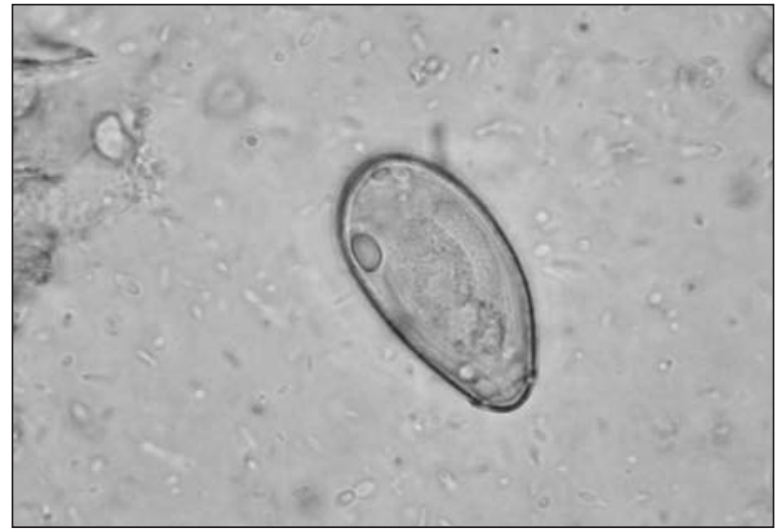

Figura I. Uovo di Opisthorchis felineus (da feci umane)

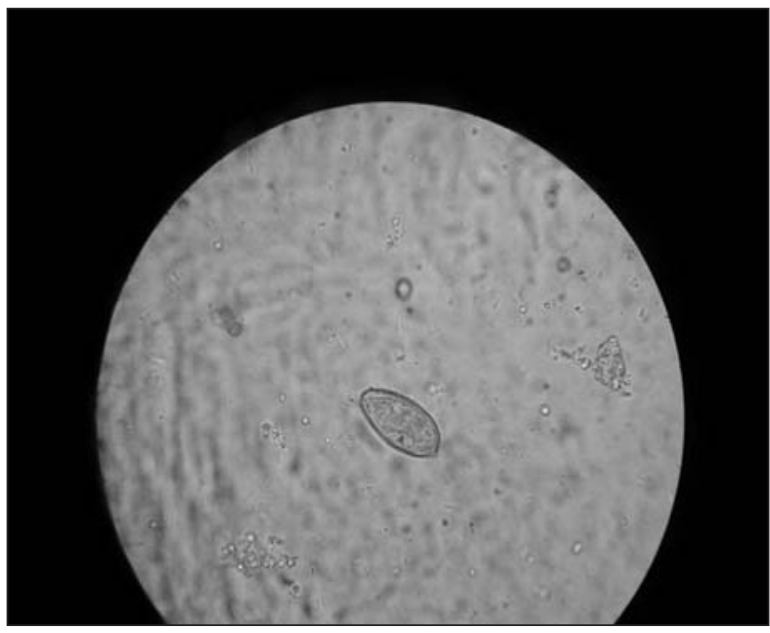

Figura II. Uovo di Opisthorchis felineus da feci di gatto 


\section{DISCUSSIONE}

Quanto osservato in tale due episodi epidemici, peraltro clinicamente silenti di opisthorchiasi, dimostra, anche se manca delle ultimissime conferme, che $O$. felineus è autoctono nel Lago Trasimeno (6). Sono in corso accertamenti su partite di pesce di provenienza dal lago (ove pescati) per la ricerca delle metacercarie di questo trematode che confermerebbero la sua presenza nel II ospite intermedio (11). Ed è anche in programma, con chi di competenza, anche una valutazione della presenza del mollusco che rappresenta il I ospite intermedio di questa infestazione zoonotica $(10,15)$. Le attente indagini epidemiologiche hanno infatti confermato l'assoluta attuale provenienza autoctona di tutti i pesci sopraccitati, avendo altresì escluso un consumo in tutti i soggetti coinvolti di pesce di altra provenienza nonché il suo consumo in altre aree o sedi ove l'endemicità è nota $(14,15)$.

Vogliamo concludere ribadendo ancora una volta l'interesse di quanto osservato, perché di indubbia rilevanza sia in campo umano che in campo animale e perciò stesso in ambito di sanità pubblica, verso cui bisogna prestare una maggiore attenzione. Se così fosse si imporrebbero più decise sollecitazioni a evitare di consumare pesce non adeguatamente cotto, dal momento che è l'unica o comunque la più razionale misura igienico-preventiva realizzabile. È infatti cosa improba e fors'anche del tutto inopportuna l'eliminazione del mollusco che rappresenta il I ospite intermedio. Inoltre l'importanza di un'attenta e meticolosa anamnesi è sempre inequivocabilmente utilissima per potere intraprendere un percorso diagnostico adeguato (9). Infatti, la disconoscenza della problematica potrebbe sottostimare tale parassitosi, in virtù anche delle caratteristiche morfologiche, e prima di tutte la ridotta dimensione, delle uova di tale platelminta, in conseguenza delle quali anche ad un occhio non inesperto (ma non adeguatamente consapevole), tale diagnostica potrebbe sfuggire (16). Va da sé che qualsivoglia conoscenza epidemiologica di un problema di e in sanità pubblica ed in ambito di zoonosi sostenute da microrganismi o macroorganismi, quali sono gli elminti in particolare, non può che essere basata sul supporto di una razionale, adeguata e responsabile capacità e quindi bontà diagnostica; ecco quindi la necessità di idonee risorse diagnostiche al riguardo e, prima ancora, di un approccio intelligente e cosciente all'attività routinaria di un laboratorio di microbiologia e parassitologia clinica.

\section{RINGRAZIAMENTI}

Gli Autori ringraziano la dottoressa M. C. Medori, dell'Azienda Ospedaliera di Terni, e la dottoressa R. Papili, dell'Azienda Ospedaliera di Perugia, per il supporto diagnostico fornito.

\section{BIBLIOGRAFIA}

1. Agaoglu A, Berktas M, Akkan H, Ceylan E. Opistorchis felineus in a Van Cat: Case Report. Y. Y. Vet. Fak. Derg. 2000; 11: 65-66.

2. Ash L, Orihel T. Liver Flukes: Clonorchis sinensis, Opisthorchis species, and Dicrocoelium dendriticum; in: Atlas of Human Parasitology. ASCP Press, Chicago, IV Edition, 1997; 289-311.

3. Bernieri F, Crotti D, Galli D, Raglio A. Elmintosi umane dell'apparato gastro-enterico esotiche, rae o eccezionali in Italia. Microbiol. Med. 2002; 17: 378386.

4. Bernieri F, Crotti D, Galli D, Raglio A. Manuale illustrato di diagnostica parassitologica, Edizioni Selecta Medica, Pavia, 2001.

5. Crotti D, D'Annibale ML, Fonzo G, et al. Dientamoeba fragilis is more prevalent than Giardia duodenalis in children and adults attending a day care centre in Central Italy. Parasite 2005; 12: 165-170.

6. Crotti D, Fioravanti ML, Gustinelli A, Florio D, Pampiglione S. Two cases of human Opisthorchiasis in Italy. Parassitologia ; 46 (Suppl. 1): 111.

7. De Carneri I. Parassitologia generale e umana. Casa Editrice Ambrosiana, Milano, XI Edizione, 1992.

8. Faust EC, Russell PF, Jung RL Opistorchis felineus; in: Clinical Parasitology, Lea \& Febiger, Philadelphia, VIII Edition, 1970; 208-211.

9. Garcia LS. Liver and Lung Trematodes; in: Diagnostic Medical Parasitology, ASM Press, Washington D. C., IV Edition, 2001; 424-433.

10. Lloy S, Soulsby EJ. L. Other Trematode Infections; in: Zoonoses, Edited by S. R. Palmer, Lord Soulsby and D. I. H. Simpson, Oxford University Press, Oxford, 1998; 731-746.

11. Pampiglione S, Canestri Trotti G. Trematodi epatici; in: Guida allo studio delle Parassitologia, Casa Editrice Esculapio, Bologna, II Edizione, 1999; 142148.

12. Perroncito E. I parassiti dell'uomo e degli animali utili, Vallardi, Milano, 1902.

13. Rivolta G. Distoma felineum. G. Anat. Fisiol. Patol. Animal. 1886; 16: 20-28.

14. Tselepatiotis E, Mantadakis E, Paoulis S, et al. A case of Opisthorchis felineus in a pilot from Greece. Infection 2003; 31: 430-432.

15. Yossepowitch O, Gotesman T, Assous M, et al. Opisthorchiasis from imported raw fish. EID 2004; 10: 2122-2126.

16. Zeibig EA. Clinical Parasitology. A practical approach. WB. B. Saunders Company, Philadelphia, 1997.

Daniele Crotti,

Strada Comunale per Pilonico Paterno 4

06080 Pianello, Perugia

Tel.: 075602372

E-mail: nenedc@tin.it 